\title{
Hierarchial Coassembly of a Cyanine Dye in Poly(vinyl alcohol) Fibrous Films by Electrospinning
}

\author{
Mustafa M. Demir, ${ }^{*}{ }^{\dagger}, \S$ Nesrin Horzum, ${ }^{\dagger}$ Bengisu Özen, ${ }^{\dagger}$ and Serdar Özçelik ${ }^{*}{ }^{\dagger}$ \\ ${ }^{\dagger}$ Department of Chemistry, and ${ }^{\S}$ Department of Material Science and Engineering, İzmir Institute of Technology, Urla, 35430 İzmir, \\ Turkey
}

Supporting Information

ABSTRACT: We report molecular aggregate formation of TTBC $\left(1,1^{\prime}, 3,3^{\prime}\right.$-tetraethyl$5,5^{\prime}, 6,6^{\prime}$-tetrachlorobenzimidazolocarbocyanine) in submicrometer-sized PVA (poly(vinyl alcohol)) fibers by electrospinning. The formation of the molecular aggregate is examined by solution and instrumental parameters of electrospinning. The precursor solution of PVA/TTBC, in the range of $0.016-0.065$ wt $\%$ is subjected to electrospinning under an electrical field ranging from 0.95 to $1.81 \mathrm{kV} \mathrm{cm}{ }^{-1}$. Both randomly deposited and uniaxially aligned fibers are achieved by using two parallelpositioned metal strips as counter electrode. Photoluminescence and polarized Fourier transform infrared spectroscopies are employed to determine spectral properties of the fibers. H-aggregates are formed within the electrospun fibers, regardless of their alignment, and $\mathrm{H}$ - and J-type aggregates coexist in the alternative spin-coated and the cast films. A strongly polarized photoluminescence emission is observed in the direction of uniaxially aligned fibers as a result of the orientation of the H-aggregates along the fiber axis. We demonstrate that electrospinning is a process capable of forming and orienting TTBC aggregates during the structural development of the polymer/dye nanofibers. These fibrous films may potentially find applications in optics and electronics.

\section{INTRODUCTION}

Molecular aggregates of dyes have attracted remarkable interest because they exhibit collective optical/excitonic properties with various functions. The strong coupling of transition dipoles of the constituent dye molecules which have internal molecular arrangement within the aggregate leads to delocalized excitonic states over a few to several molecules and, accordingly, the enhancement of the optical properties. ${ }^{1}$ The aggregation patterns of the dye molecules exhibit red shift (J-type) and blue shift (H-type) in absorption bands. ${ }^{2,3}$ TTBC is a twodimensional planar molecule that can form secondary interactions, yielding intriguing supramolecular structure. This cyanine dye undergoes both $\mathrm{H}$ - and J- type aggregation by shortrange noncovalent interactions, such as dipole-dipole interactions, $\pi-\pi$ stacking, and hydrogen bonding. Aggregation is driven mainly by the presence of a strong transition dipole moment formed by conjugated $\pi$ - electrons of the polymethine backbone of cyanine dyes. ${ }^{4}$ The construction of sophisticated molecular dye assemblies and highly ordered dye aggregates plays a significant role in designing smart materials because their optical properties show alteration depending on the structural organization of the dyes. ${ }^{5}$

The understanding of internal organization and controlling the mesoscopic morphology of cyanine aggregates has provided a powerful way of developing techniques for fabrication of functional structures designed for specific purposes. To date, a number of approaches have been demonstrated to form and align the aggregates, such as magnetic field, ${ }^{6-9}$ vertical spin coating, ${ }^{10-12}$ Langmuir-Blodgett films, ${ }^{13,14}$ adsorption on a single crystal surface, ${ }^{15,16}$ anisotropic evaporation of solution, ${ }^{17,18}$ rubbing, ${ }^{19}$ and electrospinning. ${ }^{20}$ Among these techniques, electrospinning has been proven to be a simple and efficient process to produce coassembly of highly ordered molecular aggregates induced by strong electrical field within submicrometer-scale fibers. The potential difference applied during the process requires continuous stretch of the polymer chain at the molecular level. The spatial alignment of electrospun fibers can be controlled by using a collector consisting of two parallelpositioned metal plates separated by an air gap, ${ }^{21}$ a drum rotating at high speed, ${ }^{22}$ a wheel-like bobbin, ${ }^{23}$ etc. Another fascinating feature of the process is that it can be applied to a wide variety of polymer solutions containing 1D nanostructured constituent units, such as nanowires, ${ }^{24}$ nanotubes, ${ }^{25}$ nanorods, ${ }^{26}$ etc.

Since electrospinning is based on stretching of solution jet from a charged apex through the grounded electrode, it allows the assembly of the molecular aggregates and directs the resulting orientation of the assembly to the jet axis. This process is recognized as a practical and efficient way for the fabrication of 1D molecular structure. The combination of supramolecular chemistry (molecular aggregates) and electrospinning may provide solutions for design and development of nanoscale structures. For instance, Kuo et al. ${ }^{27}$ studied electrospinning of [2,7-(9,9-dihexylfluorene)]-block-poly(methyl methacrylate) (PF-b-PMMA) which are rod-coil block copolymers. The

Received: May 21, 2013

Revised: August 5, 2013

Published: August 20, 2013 
authors reported that different aggregated structures of PF block (dotlike, linelike, and ellipselike) within nanofibers can be obtained by varying only the ratio of dimethyl formamide (DMF)/tetrahydrofuran (THF) solvents of precursor electrospinning solutions. The resulting electrospun fibers with several morphologies lead to particular photophysical properties through the use of selective solvent. Kalra et al. ${ }^{28}$ showed microphase separation as cylindrical and lamellar morphology in electrospun poly(styrene-block-isoprene) (PS- $b$-PI) nanofibers by systematically controlling the block copolymer composition, the length, and shape and distribution of these worm-like entities. One dimensional arrangement of the gold particle array within submicrometer poly(ethylene oxide) fibers was fabricated by Kim et al. ${ }^{29}$ to use for future nanodevices.

Recent studies show synergistic efforts of both bottom-up and top-down approaches to form fine hierarchical nanostructures. In our previous report, ${ }^{20}$ we combined the formation of J-aggregate and electrospinning processes using a pseudoisocyanine $\left(1,1^{\prime}\right.$ diethyl-2,2'-cyanine bromide, PIC) and poly(vinyl alcohol) (PVA) system in aqueous alcoholic solutions. PIC is one of the frequently used cyanine dyes that form only a one-dimensional fibrous-like J-aggregate. Although PIC molecules were individually dispersed in the precursor solution, directed assembly of Jaggregates was induced by electrospinning process. Electrospinning not only provided the formation of J-aggregates but also allowed the orientation of the J-aggregates along the fiber axis. In the present study, using the same polymer/solution system, we employed another cyanine dye, TTBC, which facilitates formation of two different types of aggregates in terms of molecular arrangement. In this work, we report the formation of highly oriented $\mathrm{H}$-aggregates of TTBC dye within the aligned electrospun PVA fibers.

\section{MATERIALS AND METHODS}

Chemicals. Poly(vinyl alcohol) $\left(M_{\mathrm{w}}\right.$ of $30-70 \mathrm{~kg} \mathrm{~mol}^{-1}$, Sigma-Aldrich, 87-90\% hydrolyzed), 1,1',3,3'-tetraethyl5,5',6,6'-tetrachlorobenzimidazolocarbocyanineiodide (TTBC) (Hayashibara Biochemical Company), methanol (Sigma-Aldrich, $\geq 99.8 \%$ ), and sodium hydroxide (Sigma-Aldrich, $\geq 97.0 \%)$ were used as received without further purification. Deionized water $(18.2 \mathrm{M} \Omega \mathrm{cm})$ was used throughout the study.

Preparation of TTBC/PVA Films. The precursor solution of TTBC/PVA system was prepared as follows: A $25 \mathrm{mM}$ stock solution of the cyanine dye was prepared by dissolving the TTBC in methanol. TTBC solutions in the concentration range of 0.025-1.0 mM were prepared by an appropriate dilution of the stock solutions. Aqueous solutions of $10 \mathrm{wt} \%$ PVA were prepared by stirring and heating the solution to $100{ }^{\circ} \mathrm{C}$ until complete dissolution. A homogeneous solution including a desired amount of TTBC in $1 \mathrm{~mL}$ of methanol was added dropwise into $1 \mathrm{~g}$ of PVA solution (7.5-15.0 wt \%) under stirring. The methanol fraction of the solution was evaporated, and the resulting aqueous dye/polymer solution was subjected to electrospinning at $35{ }^{\circ} \mathrm{C}$ in a horizontal direction in a glass chamber. As a counter electrode, the two parallel-positioned metal strips with a $1.0 \mathrm{~mm}$ void gap was used. The thickness of the metal strip is $105 \mu \mathrm{m}$. The strips get sharper nearer the edges, and the thickness of the sharp edges is $90 \mu \mathrm{m}$. The tip-tocollector distance was $6 \mathrm{~cm}$, and the flow of the solutions was held constant at $0.5 \mathrm{~mL} \mathrm{~h}^{-1}$ using a syringe pump (LION WZ$50 \mathrm{C6}$ ). The applied voltage was varied from 6.7 to $12.7 \mathrm{kV}$.

Thin films were prepared by spin-coating and film-casting for comparison. The spin-coated films were prepared by using a WS
400B 6NPP/LITE spin coater. One milliliter of TTBC (0.5 $\mathrm{mM}) /$ PVA (10 wt \%) solution was placed on the glass substrate, which was then rotated at 1000, 2500, 5000, and $8000 \mathrm{rpm}$ for 1 $\mathrm{min}$. The cast films were prepared by dropping $0.5 \mathrm{~mL}$ of TTBC $(0.5 \mathrm{mM}) /$ PVA $(10 \mathrm{wt} \%)$ solution on to the glass substrate. The films were left for evaporation of solvent in a dark environment.

Fabrication of Polymer-Free TTBC Fibers. The solution of J-aggregates was prepared by stirring $1 \mathrm{~mL}$ of $1.0 \mathrm{mM}$ TTBC in methanol with $3 \mathrm{~mL}$ of $0.01 \mathrm{M} \mathrm{NaOH}$ aqueous solution. During the electrospinning, the flow of dispersion was held constant at $0.5 \mathrm{~mL} \mathrm{~h}^{-1}$ using the syringe pump. The applied voltage was $12.7 \mathrm{kV}$, and the tip-to-collector distance was set to 6 $\mathrm{cm}$. The resulting fibers were dried at $50{ }^{\circ} \mathrm{C}$ in a vacuum to ensure the removal of any residual solvent.

Characterization Methods. Absorption and photoluminescence (PL) emission spectra were obtained by a Varian Eclipse spectrofluorometer equipped with polarizers. For morphological observations, polarized optical microscopy was carried out with an Olympus BX51 microscope, and scanning electron microscopy (SEM) was performed on a Phillips XL-30S FEG. The fiber diameter distribution analysis from SEM micrographs was examined with the Image J program. Fourier transform infrared (FTIR) spectra were registered with a PerkinElmer Spectrum 100 spectrometer. The polarization of the incident IR radiation was achieved with a $\mathrm{ZnSe}$ polarizer.

\section{RESULTS AND DISCUSSION}

Structural and Spectral Characterization. The aim of this work was to control the type of TTBC aggregation by varying chemical (solution) and physical (instrumental) parameters of electrospinning process. A set of TTBC/PVA solutions were prepared and subjected to electrospinning under different applied voltages. Figure 1 shows the chemical structure of TTBC and absorption spectra of the dye in aqueous PVA solutions. The spectra of various concentrations ranging from 0.025 to $0.500 \mathrm{mM}$ exhibit the absorption band of TTBC monomer in PVA at $520 \mathrm{~nm}$ and fluorescence band at $550 \mathrm{~nm}$. These findings are in agreement with the reported solvatochromism observed for TTBC monomer in various solvents. ${ }^{30}$ It is confirmed that TTBC molecules were molecularly dispersed in PVA solution, and the spinning solution was initially free of aggregates.

Both random and aligned TTBC/PVA fibers were obtained in single-step electrospinning using parallel positioned metal strips with an air gap (panel a of Figure 2). An overview of fibers collected on and between the metal strips was imaged by SEM. Panels $b$ and $c$ of Figure 2 demonstrated the deposition of the random and the aligned fibers, respectively. The composition of TTBA and PVA varied in precursor electrospinning solution. The diameter of the resulting fibers was examined. Panel $\mathrm{d}$ of Figure 2 shows the average fiber diameter (AFD) at various TTBC concentrations ranging from 0.025 to $0.5 \mathrm{mM}$. PVA content in solution and the applied potential difference were held constant at 10 wt $\%$ and $12.7 \mathrm{kV}$, respectively. Increasing the TTBC concentration in precursor solution did not show a remarkable increase in AFD $(\sim 100 \mathrm{~nm})$, at least in the applied concentration range. Figure 2 also shows that the aligned fibers are systematically thinner than the one of the random fibers.

When two parallel positioned metal strips were used as a counter electrode, two fiber populations were obtained. The first population is observed on the surface of the strips, where the fibers were randomly deposited. The second population is obtained across the air gap; the fibers are positioned 
(a)<smiles>CCN1C(=CC#Cc2n(C)c3cc(Cl)c(Cl)cc3[n+]2CC)N(C)c2cc(Cl)c(Cl)cc21</smiles>

(b)

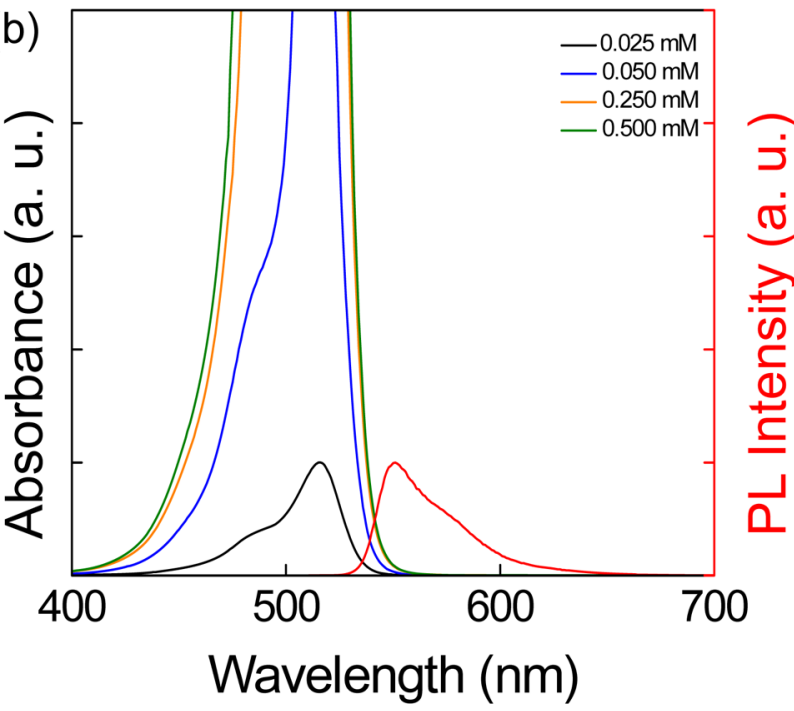

Figure 1. (a) Cationic structure of TTBC. (b) Absorption and photoluminescence spectra of individual TTBC molecules in aqueous PVA solutions at different concentrations.

perpendicular to the edge of the strips and parallel to each other. Using a sharp strip as counter electrode enables alignment of the fibers across the gap.

The electrical field applied in this process is inversely proportional to the thickness of the edge of the strip so that the intense local electrical fields are generated at close proximity of the sharp edge. ${ }^{31}$ The trajectory of the jet takes place from one edge to the other one. ${ }^{21}$ Therefore, the jet is exposed to further stretching between the gap, and the fibers accumulated between the strips were found to be thinner than the randomly deposited ones on the strips. Note that the aligned fibers deposited across the gap were not supported by the substrate. Panel e shows the effect of PVA content on the diameter of the TTBC/PVA fibers. TTBC concentration was kept constant at $1.0 \mathrm{mM}$, and PVA content was varied from 15 to $20 \mathrm{wt} \%$. The AFD was observed to be larger when PVA concentration was increased. This result is not surprising because as the polymer concentration increases, the viscosity of solution increases and extensive polymer chain entanglements take place. Hence, the charged jet is prevented from breaking up into smaller jets, and the fibers that are produced at high polymer concentrations appear to be thick. ${ }^{32,33}$ SEM micrographs of the randomly deposited and the aligned fibers with respect to the varying TTBC and PVA concentrations are given in Figures S1-S2, Supporting Information (SI).

The alignment of fibers with aggregates was confirmed by polarized optical microscopy. Figure 3 represents the polarized microscope images of the aligned TTBC/PVA fibers at different polarization angles. The images were captured at the same field of a sample with different polarization angles, that is, by varying the angle of the analyzer-polarizer. Changing the angle allowed us to observe perfectly aligned or randomly deposited fiber layers separately. The aligned fiber layer was observed when the polarization angle of the analyzer was $20^{\circ}$.
Absorption spectra of the dye solution and of the fibrous samples were obtained and are shown in Figure 4, supporting that the $\mathrm{H}$-aggregation absorption band was blue-shifted with respect to the monomer. TTBC $(0.025 \mathrm{mM}) /$ PVA solution exhibited monomer absorbance at $520 \mathrm{~nm}$. For the electrospun TTBC/PVA fibers, a new blue-shifted H-band appeared at 500 $\mathrm{nm}$ with tail broadening. The hypsochromic shift in the absorption spectrum was ascribed to the formation of $\mathrm{H}$ aggregates within the fibers.

The emission spectra of the dye molecules within electrospun fibers were examined by photoluminescence (PL) spectroscopy. The spectra were normalized with respect to the monomers to follow the extent of aggregation. Panels a and b of Figure 5 show the normalized emission spectra of randomly deposited and aligned PVA/TTBC fibers electrospun at different TTBC concentrations. There are two emission bands observed at 485 and $535 \mathrm{~nm}$, corresponding to the H-band and monomer band, respectively. ${ }^{10}$ These assignments are, moreover, supported by the absorption spectra of the $\mathrm{H}$-aggregates in the electrospun fibers. It was demonstrated that $\mathrm{H}$-aggregates and a small amount of monomers exists in both the random and the aligned fibers. No J-aggregate formation was observed. Moreover, the emission of $\mathrm{H}$-band generally could not be observed in solutions or interfaces, but in this particular case, the $\mathrm{H}$-aggregate, which was formed in a concentrated polymeric media and confined to onedimensional growth of PVA fibers, emits in blue region. Furthermore, the increase in TTBC concentration resulted in a small decrease of $\mathrm{H}$-band intensity with respect to the monomer. At higher TTBC concentrations, the applied electrical force during the process is not enough to form more $\mathrm{H}$-aggregates. In addition, comparison of emission spectra of the random and the aligned fibers indicates that the aligned fibers have higher $\mathrm{H}$-band intensity than the one in random fibers with respect to the monomer intensity. This result can be correlated with the higher electrical field that the aligned fibers are exposed during the process. The formation of the $\mathrm{H}$-type aggregates rather than the J-type is a result of parallel orientation of TTBC molecules favoring the $\mathrm{H}$-aggregates alone. Since a very high electrical field is applied, it forces TTBC molecules to be arranged parallel to the field and forming the $\mathrm{H}$-aggregates in the electrospinning processes.

The PL spectrum of the electrospun TTBC/PVA shows a monomer emission band at $535 \mathrm{~nm}$. Previously, it was shown that the PL band positioned around $550 \mathrm{~nm}$ was attributed to the monomeric TTBC molecules. The monomer band in the TTBC/PVA solution was blue-shifted compared with the monomer band in the electrospun fibers, probably as a result of the solvatochromic effects based on environmental change from solution to the solid phase. Halpert and co-workers ${ }^{34}$ reported a blue shift of the J-band of TTBC molecules associated with $\mathrm{CdSe} / \mathrm{ZnS}$ quantum dots (QD) with respect to that of aggregated TTBC in the absence of QDs. This difference was likely to be attributed to the size of the aggregates formed on the QDs and solvatochromic effects.

Figure 6 shows photoluminescence (PL) emission spectra of electrospun TTBC/PVA fibers prepared at different PVA contents. The aim of varying the PVA content was to investigate the effect of the fiber diameter on the $\mathrm{H}$-aggregate formation. The PL spectra of the different diameter fibers remain almost unchanged. As a result, the fiber diameter was found to have no effect on the aggregation process, at least in the range of concentration we employed. In addition, an increased amount of 


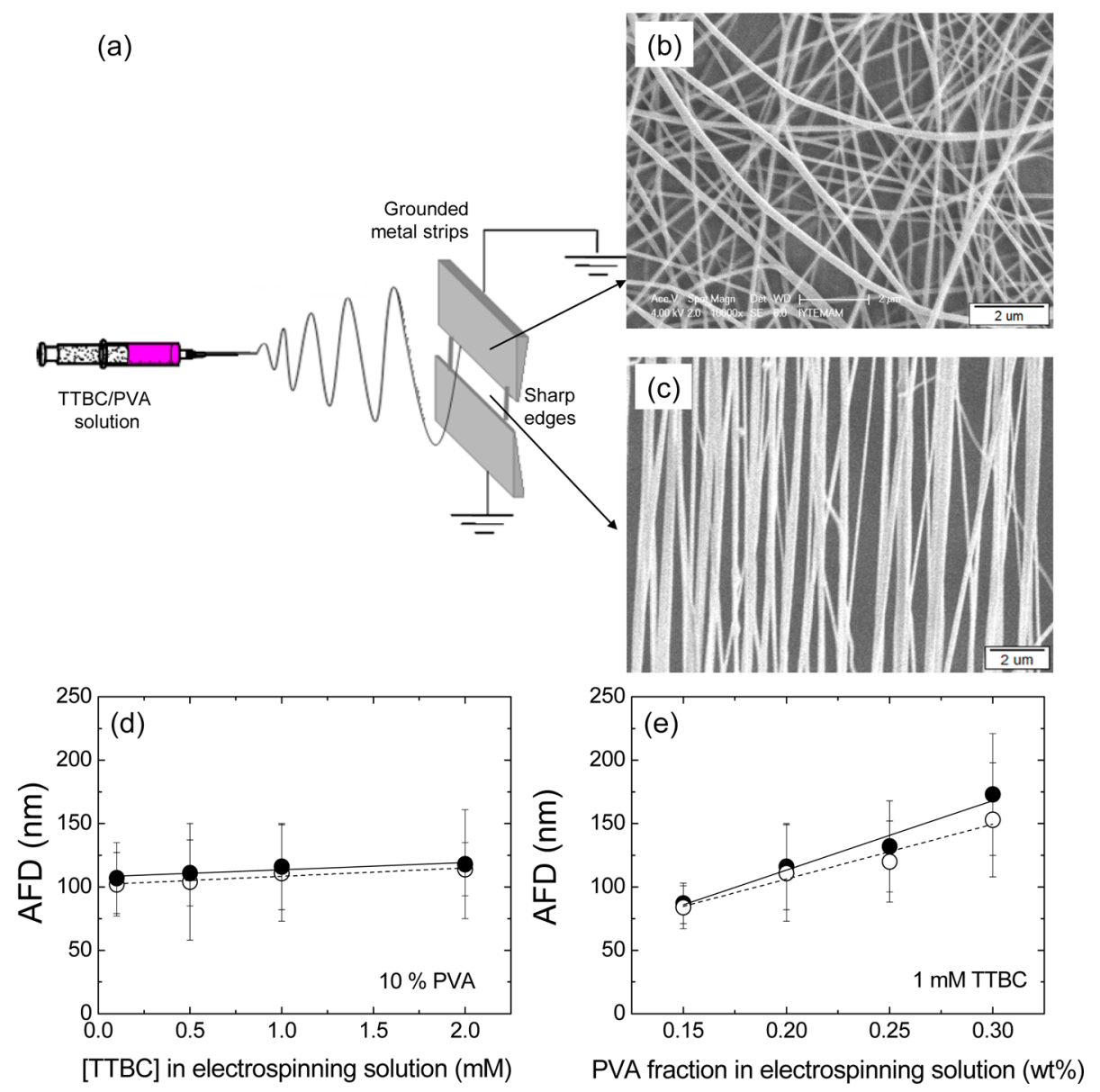

Figure 2. (a) Schematic representation of experimental set up. SEM micrographs of (b) randomly deposited electrospun fibers collected on the surface of metal strips, (c) aligned fibers uniaxially across the void gap. Microscopy reveals the formation of the random and the aligned populations of fibers. Average fiber diameter (AFD) at different (d) TTBC concentrations and (e) PVA fractions for (O) random (O) aligned fibers. The concentration of TTBC has a minor effect on AFD; however, the main parameter for the fiber diameter seems to be the concentration of PVA.
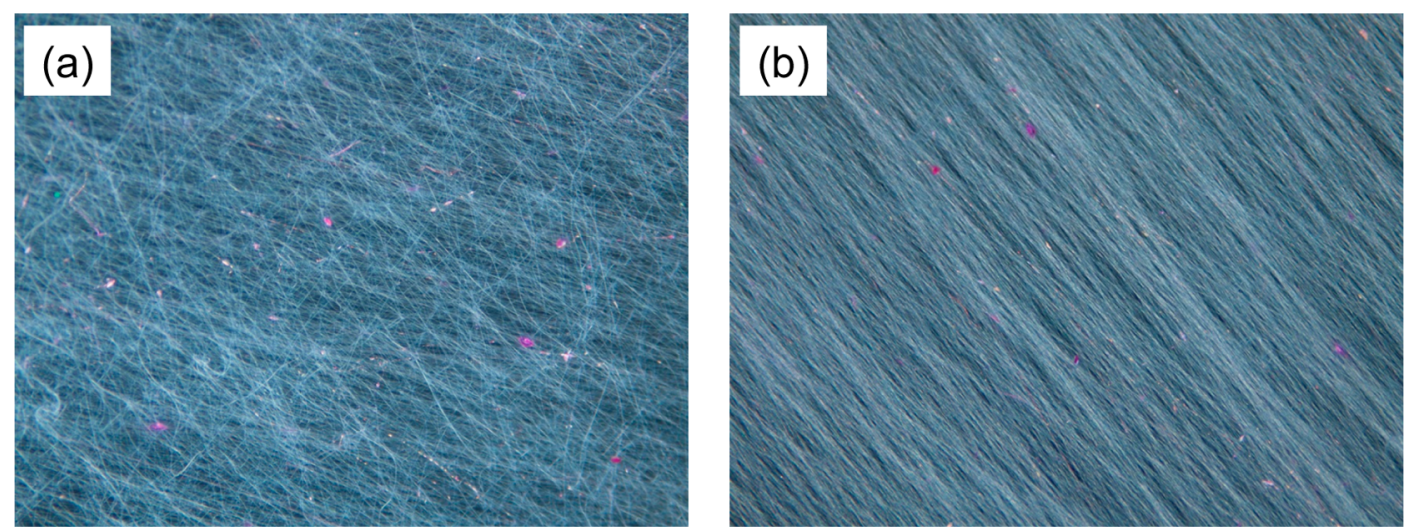

Figure 3. Polarized microscope images of TTBC $(0.5 \mathrm{mM})$-doped PVA fibers $(10 \mathrm{wt} \%)$ performed at different polarization angles of $(\mathrm{a}) 0^{\circ}$ and $(\mathrm{b}) 20^{\circ}$. The aligned fiber layer is obtained when the angle is rotated $20^{\circ}$. Note that PVA fibers without TTBC do not show aligned morphology under a polarized microscope at this angle.

PVA yielded an increase in the signal level of monomeric emission, confirming the consistency of the spectral assignments.

The PL emission spectra of the resultant fibers under various potential differences applied are shown in Figure 7. There are bands corresponding to the $\mathrm{H}$-aggregates and monomers within fibers, respectively. However, an emission due to J-aggregate formation was not observed. The increment in potential difference enhances the emission intensity of the $\mathrm{H}$-aggregates.
The reason for this enhancement is that the applied potential difference in electrospinning process exerts an electrical force on the surface of the droplet, in addition to an electrical field between the tip of the needle and the collector. As the applied potential difference increases, the strength of electrical force acting on TTBC molecules increases. These physical processes direct TTBC monomers to assemble and align themselves 


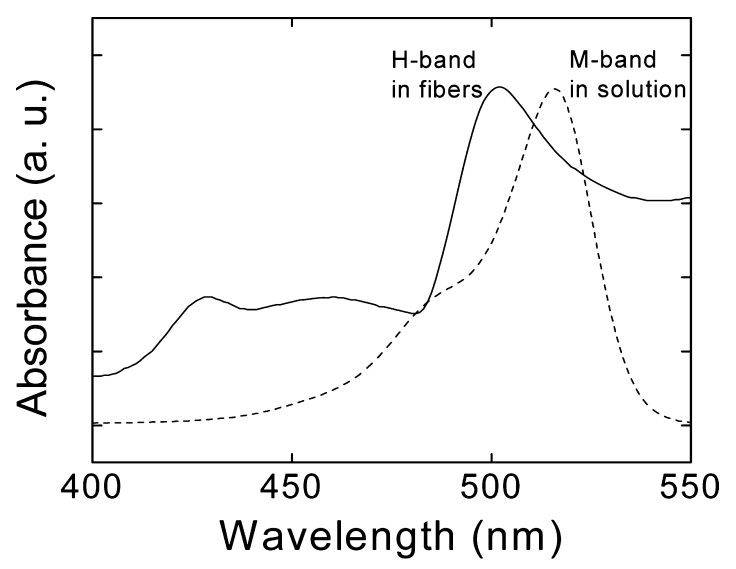

Figure 4. Absorption spectra of the TTBC $(0.025 \mathrm{mM})$ in PVA solution and the TTBC/PVA fibers.

(a)

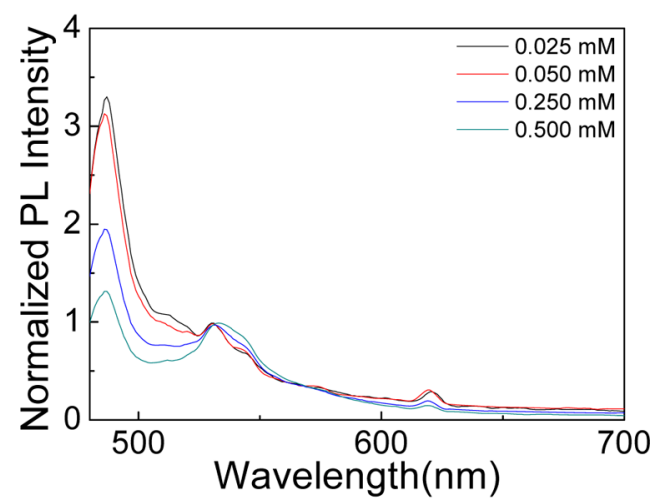

(b)

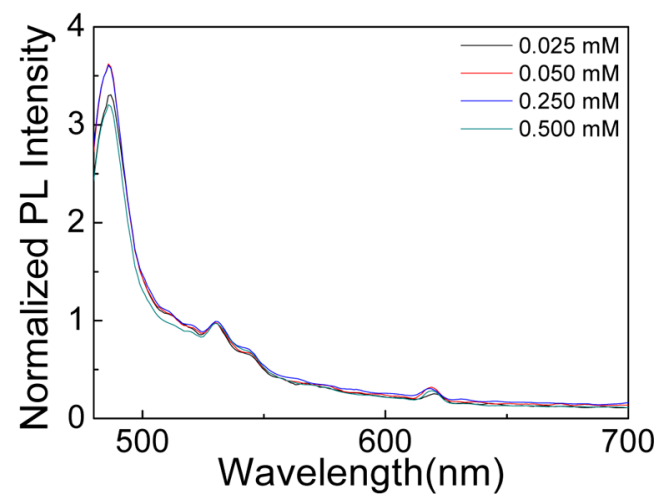

Figure 5. PL spectra of TTBC/PVA (a) the randomly deposited and (b) the aligned fibers prepared at different TTBC concentrations. An increase in TTBC concentration results in a decrease in the H-band intensity with respect to the monomer.

parallel to the direction of electrical field during the electrospinning.

Polarized FTIR spectroscopy was used to analyze the effect of applied potential difference and TTBC concentration on the orientation of PVA chains. Therefore, the polarized FTIR spectra of the fibers electrospun at different potentials and TTBC concentrations were recorded and analyzed. The degree of orientation of the PVA chains was quantified by the dichroic ratio $\left(R=A_{\|} / A_{\perp}\right)$ that was calculated using the absorbance values of the vibration bands of $\mathrm{CO}$ and $\mathrm{CH}_{2}$ stretching of PVA. Figure 8 shows the polarized FTIR spectra of the aligned TTBC/PVA fibers and the calculated dichroic ratios with respect to the applied potential. For each spectrum, the dichroic ratio of PVA

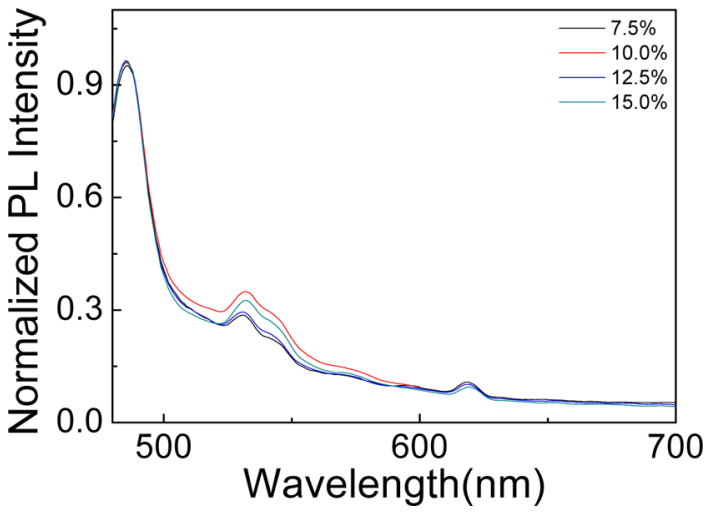

Figure 6. PL spectra of electrospun TTBC/PVA fibers prepared at various PVA contents $(7.5,10.0,12.5,15.0$ wt \%). It seems that the diameter of fiber has no effect on TTBC aggregate formation.

(a)

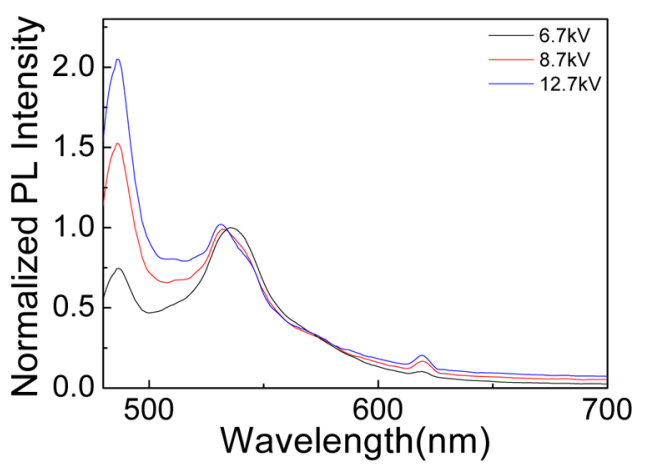

(b)

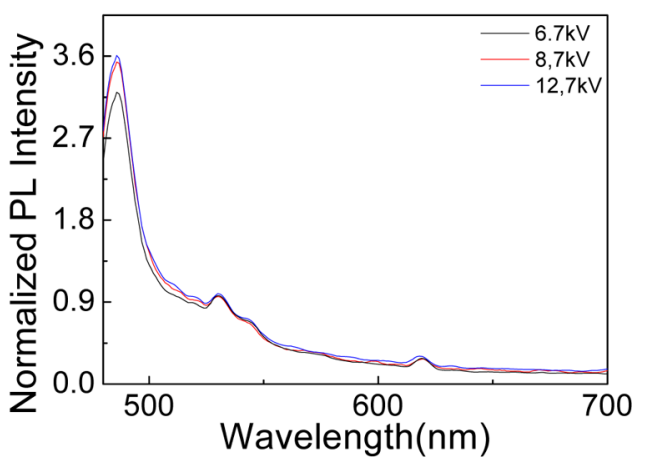

Figure 7. PL spectra of (a) randomly deposited and (b) aligned fibers electrospun from TTBC $(0.5 \mathrm{mM}) / \mathrm{PVA}(10 \mathrm{wt} \%)$ at different potential differences. The increase in potential difference enhances the emission intensity of $\mathrm{H}$-aggregates.

chains was determined with respect to the signals at 1097 (CO) and $2942 \mathrm{~cm}^{-1}\left(\mathrm{CH}_{2}\right)$. For the aligned fibers, the dichroic ratio was found to be $\sim 3$, proving the chain alignment parallel to the fiber axis. The resultant values showing the effect of TTBC concentration and potential difference on the alignment of fibers are illustrated in panel b of Figure 8. Increasing TTBC concentration results in higher chain orientation of PVA fibers. This finding may point out the ionic character of TTBC/PVA solution. Higher ion concentration induces strong electric fields at a constant applied potential difference. The more electric field acts on polymer chains; a higher orientation of the chains is induced in the growth direction of the aligned fibers.

The orientation of the $\mathrm{H}$-aggregates within the fibers of TTBC/PVA system was studied by polarized PL spectroscopy 


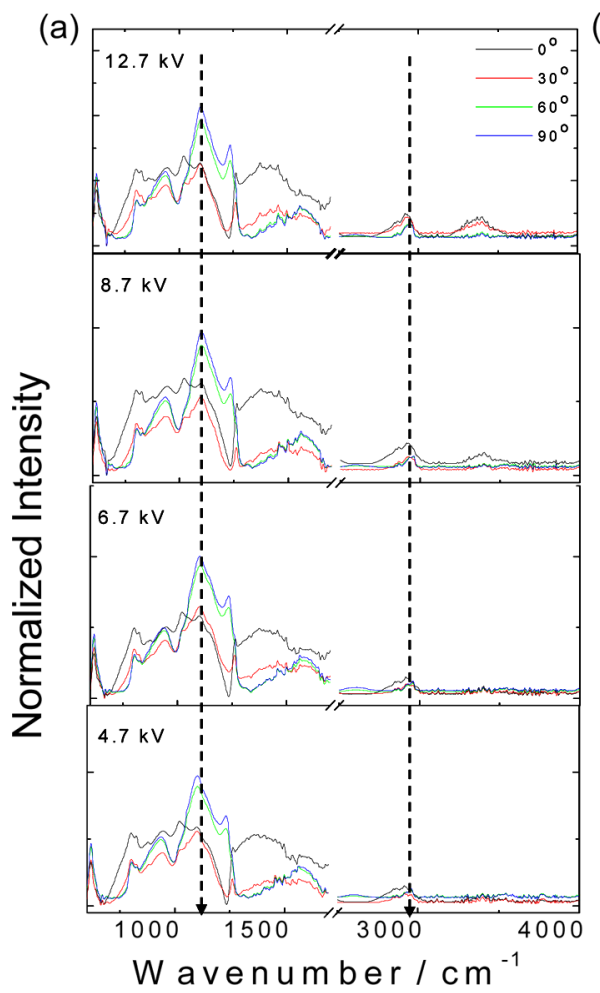

(b)

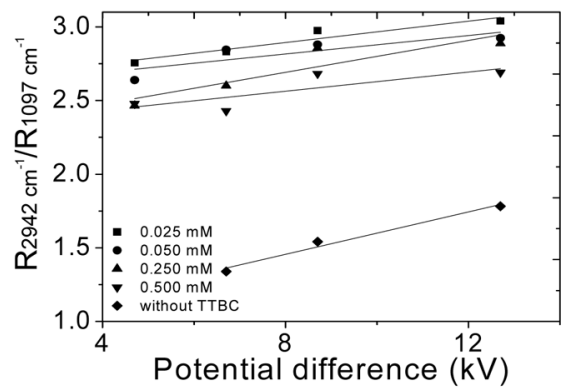

Figure 8. (a) Polarized FTIR spectra for aligned TTBC/PVA fibers obtained at different potential differences. (b) Dichroic ratio versus potential difference plot, as derived from polarized FTIR spectra. This result shows the orientation of PVA chains to the fiber axis.

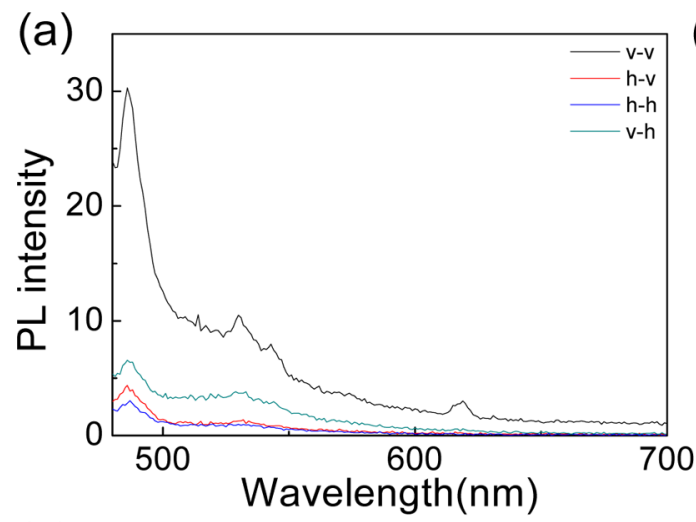

(c)
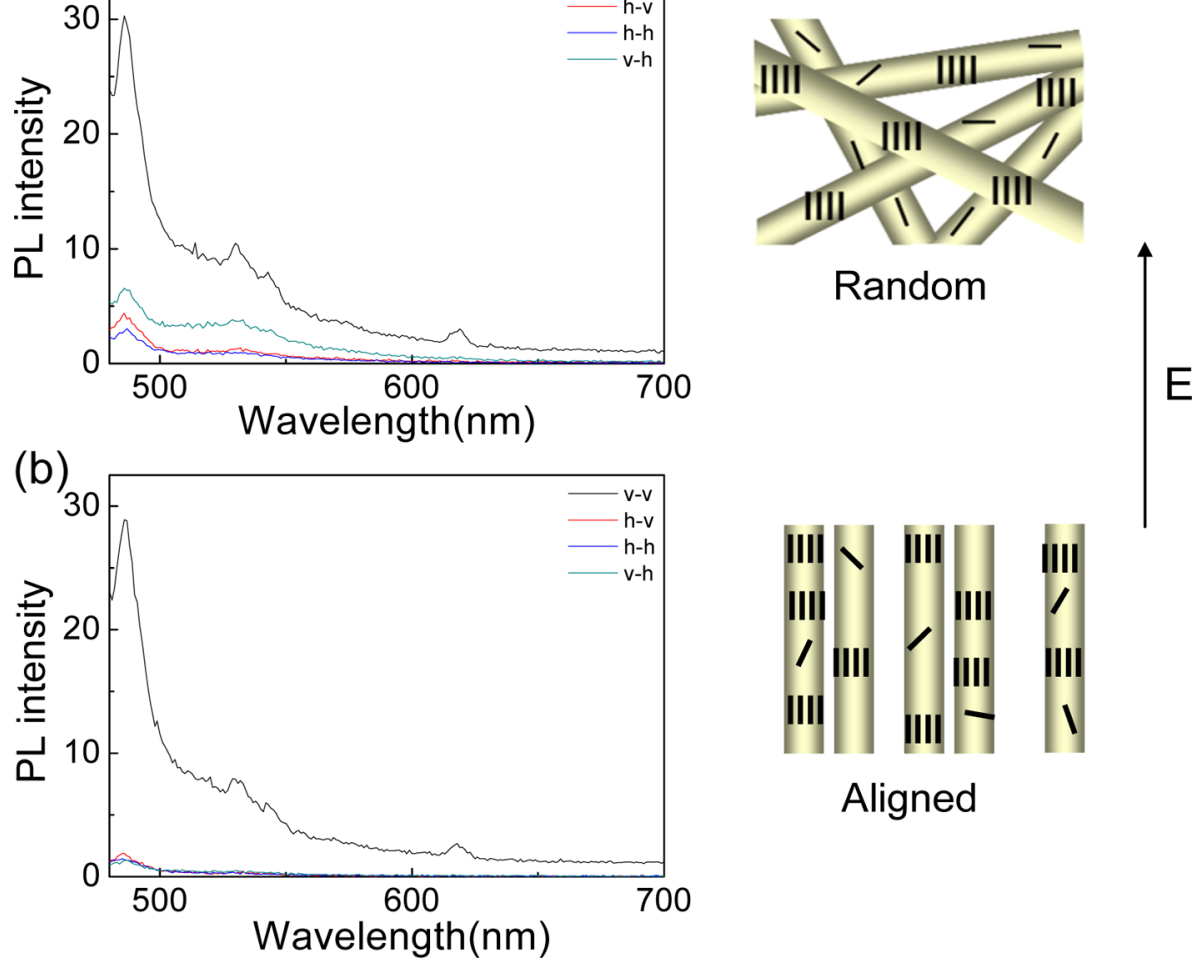

Aligned

Figure 9. Polarized PL spectrum of (a) random and (b) aligned fibers of the TTBC (0.5 mM)/PVA (10 wt \%) system performed at four different polarizer combinations that are vertical-vertical $(\mathrm{v}-\mathrm{v})$, vertical-horizontal $(\mathrm{v}-\mathrm{h})$, horizontal-horizontal $(\mathrm{h}-\mathrm{h})$, and horizontal-vertical $(\mathrm{h}-\mathrm{v})$ with respect to the fiber axis. (c) Schematic representation of the orientation of $\mathrm{H}$-aggregates under electrical field. The formation and orientation of $\mathrm{H}$-type TTBC aggregates with respect to fiber axis are shown. 
(Figure 9). A strong polarized emission was observed when light was polarized parallel to the aligned fiber axis. The intensity decreased remarkably for the other three polarizations (panels a and $b$ of Figure 9). This polarization dependence indicates that the $\mathrm{H}$-aggregates aligned along the fiber axis. Surprisingly, the randomly deposited fibers also showed polarization dependence.

For reasons of comparison of fibrous electrospun films, two alternative thin film formation processes were employed: (i) spin-coating and (ii) film casting. The intention was to investigate the effect of electrical force applied during the process on the formation of the molecular aggregates. Figure 10

(a)

(b)
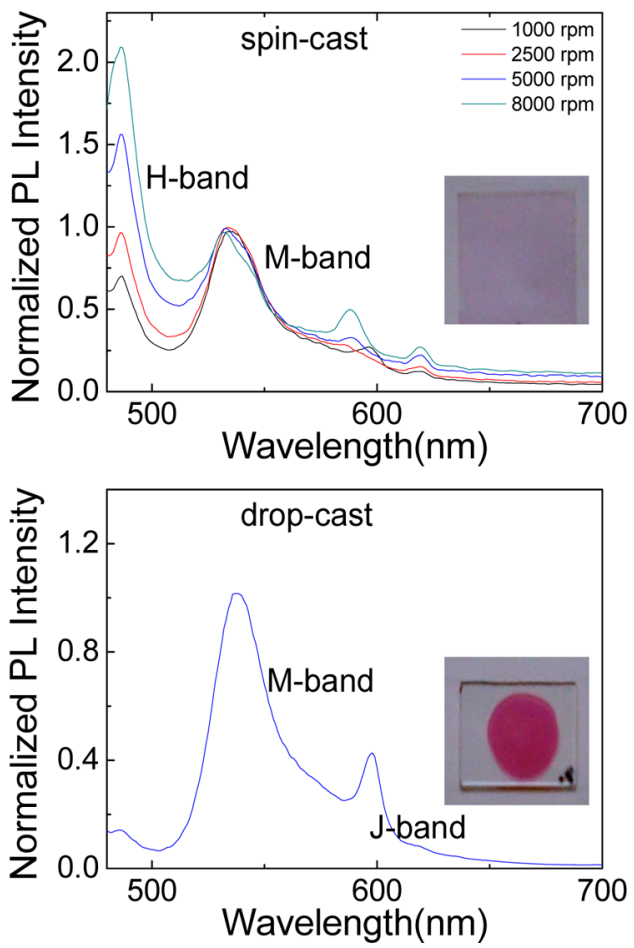

Figure 10. PL spectrum of (a) spin-coated films prepared at different rotation speeds, (b) cast film of TTBC $(0.5 \mathrm{mM}) /$ PVA (10 wt \%). In contrast to fibrous fibers obtained by electrospinning, the spin-coated films contain both $\mathrm{H}$ - and the J-aggregates, whereas the cast film initiates the formation of J-aggregates of TTBC.

shows PL spectra of TTBC/PVA films prepared by spin-coating at different rotation speeds ranging from 1000 to $8000 \mathrm{rpm}$ and by film-casting. The insets show photographs of the spin-coated and the cast films. The spin-coated films are continuous, and their thicknesses are around $2 \mu \mathrm{m}$. They contain both the $\mathrm{H}$ - and the J-aggregates as well as monomers. J-aggregates of TTBC were observed along with monomeric TTBC. On the other hand, the cast film has a heterogeneous appearance and is about $100 \mu \mathrm{m}$ thick. Furthermore, applying a higher centrifugal force during the spin-coating process induces the formation of both the $\mathrm{H}$ - and the J-aggregates with respect to the monomers.

Polymer-Free Fibers from Monomeric TTBC. In this study, we showed that TTBC monomers in PVA solutions were inevitably assembled into $\mathrm{H}$-aggregates upon electrospinning. The question arose as to whether J-aggregates already formed in solutions induce fibrillar structures when they are subjected to electrospinning in the absence of PVA, that is, without a carrier polymer matrix. Since the most important criteria for the fiberformation by electrospinning are the entanglement of long polymer chains and the viscosity of solution, high-molecularweight polymers with higher polymer concentrations result in fiber formation. On the other hand, the dye aggregates can form noncovalently bonded, long, supramolecular structures. This feature may cause the formation of fibers or fiberlike morphologies in the absence of polymer. In this sense, Jaggregates of TTBC, prepared in aqueous $\mathrm{NaOH}$ solution, were subjected to the electrospinning process.

Figure 11 shows PL spectra of the J-aggregates in solution (black curve) and the $\mathrm{H}$-aggregates in the electrospun fibers (red

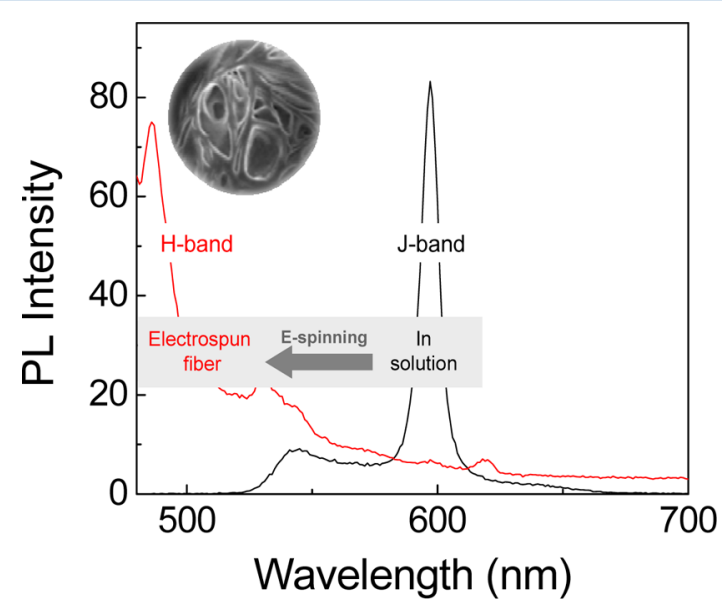

Figure 11. PL spectrum of (a) J-aggregated TTBC solution, (b) TTBC fibers electrospun from the TTBC solution. Electrospinning inevitably changes the orientation of J-aggregated TTBC molecules to the $\mathrm{H}$ aggregates.

curve). The spectra show two major bands at 599 and $488 \mathrm{~nm}$. The former one was attributed to the red-shifted J-band, and the latter one was assigned to the blue-shifted H-band upon electrospinning. This finding strongly suggests that the electrospinning process triggers the formation of $\mathrm{H}$-type of aggregates not only from monomers but also from the initially formed Jaggregates. Moreover, electrospinning is able to orient dye species, including monomer and aggregates at the molecular level through the direction of the field. The magnitude of the electric field implemented by the electrospinning should overcome the intermolecular dipolar forces induced by the J-aggregates. The strength of this interaction was found about $2100 \mathrm{~cm}^{-1} .4$ The inset SEM image reveals the formation of fiber-like TTBC structures in the absence of PVA. The presence of considerable aggregates and corresponding intermolecular interactions can effectively stabilize the electrospinning jet and therefore resulted in fibrous structure.

\section{CONCLUSIONS}

This study merges a frequently used top-down approach (electrospinning) and a bottom-up approach (formation of molecular aggregates). The effect of electrospinning on the molecular aggregation of the TTBC dye was investigated. The solution and instrumental parameters of the electrospinning were studied on a model TTBC/PVA system. Two electrospun fiber mats in terms of alignment of constituent fibers (aligned and randomly deposited) were obtained using a parallel positioned counter electrode. Independent of the alignment of the fibers, only H-type TTBC aggregates formed within electrospun PVA fibers, although initially, monomeric dispersion was present in the precursor solution. Polarized emission spectroscopy reveals 
that the aggregates within a single filament of electrospun mat are highly oriented. Consequently, a well-ordered structure was obtained using the electrospinning process at different length scales. The first one is the alignment of fibers over square millimeters, the second one is the orientation of PVA chains along the fiber axis, and the third one is the orientation of the dyeaggregates of TTBC molecules within the fibers.

The comparison of the electrospinning with other thin film preparation techniques demonstrated that electrospinning was found to be a more efficient technique to provide the formation of oriented $\mathrm{H}$-aggregates of TTBC dye. Moreover, polymer-free ultrafine TTBC fibers were successfully produced by electrospinning. Our findings revealed that the electrical field applied during the process assembles monomers into $\mathrm{H}$-aggregates and also changes the orientation of TTBC molecules in the Jaggregates, forming an $\mathrm{H}$-aggregate in which TTBC molecules are aligned parallel to each other. In the framework of nanofabrication, directed assembly of dye aggregates within electrospun fibers provides a powerful way to produce functional constructs designed for optical devices.

\section{ASSOCIATED CONTENT}

\section{S Supporting Information}

SEM micrographs of the aligned and randomly deposited TTBC/PVA fibers with respect to various TTBC and PVA concentrations. This material is available free of charge via the Internet at http://pubs.acs.org.

\section{AUTHOR INFORMATION}

\section{Corresponding Authors}

*Phone: +90 23275075 11. E-mail: mdemir@iyte.edu.tr.

*Phone: +90 23275075 02. E-mail: serdarozcelik@iyte.edu.tr.

\section{Notes}

The authors declare no competing financial interest.

\section{ACKNOWLEDGMENTS}

The authors thank Dr. Hüseyin Özgener for FTIR and UV measurements and members of IzTech-CMR for microscopy work. M.M.D. acknowledges financial support of The Scientific and Technological Research Council of Turkey (TUBITAK) career development project encoded with TBAG-107T795. S.O. thanks the State Planning Organization (DPT) of the Turkish Prime Ministry for financial support.

\section{REFERENCES}

(1) Wurthner, F.; Yao, S.; Beginn, U. Highly Ordered Merocyanine Dye Assemblies by Supramolecular Polymerization and Hierarchical Self-Organization. Angew. Chem., Int. Ed. 2003, 42, 3247-3250.

(2) Jordan, A. N.; Das, S.; Siraj, N.; de Rooy, S. L.; Li, M.; El-Zahab, B.; Chandler, L.; Baker, G. A.; Warner, I. M. Anion-Controlled Morphologies and Spectral Features of Cyanine-Based Nanogumbos - An Improved Photosensitizer. Nanoscale 2012, 4, 5031-5038.

(3) Rösch, U.; Yao, S.; Wortmann, R.; Würthner, F. Fluorescent HAggregates of Merocyanine Dyes. Angew. Chem. 2006, 118, 7184-7188.

(4) Birkan, B.; Gulen, D.; Ozcelik, S. Controlled Formation of the Two-Dimensional TTBC J-Aggregates in an Aqueous Solution. J. Phys. Chem. B 2006, 110, 10805-10813.

(5) Advincula, R. C.; Fells, E.; Park, M. K. Molecularly Ordered Low Molecular Weight Azobenzene Dyes and Polycation Alternate Multilayer Films: Aggregation, Layer Order, and Photoalignment. Chem. Mater. 2001, 13, 2870-2878.

(6) Kitahama, Y.; Kimura, Y.; Takazawa, K. Study of Internal Structure of Meso-Tetrakis(4-Sulfonatophenyl) Porphine J-Aggregates in Sol- ution by Fluorescence Microscope Imaging in a Magnetic Field. Langmuir 2006, 22, 7600-7604.

(7) Kitahama, Y.; Kimura, Y.; Takazawa, K.; Kido, G. Magnetic Alignment of Fiber-Shaped Pseudoisocyanine J Aggregates in a Gelated Solution. Bull. Chem. Soc. Jpn. 2005, 78, 727-730.

(8) Shklyarevskiy, I. O.; Boamfa, M. I.; Christianen, P. C. M.; Touhari, F.; van Kempen, H.; Deroover, G.; Callant, P.; Maan, J. C. Magnetic Field Induced Alignment of Cyanine Dye J-Aggregates. J. Chem. Phys. 2002, 116, 8407-8410.

(9) Shklyarevskiy, I. O.; Christianen, P. C. M.; Aret, E.; Meekes, H.; Vlieg, E.; Deroover, G.; Callant, P.; van Meervelt, L.; Maan, J. C. Determination of the Molecular Arrangement inside Cyanine Dye Aggregates by Magnetic Orientation. J. Phys. Chem. B 2004, 108, 16386-16391.

(10) Gulen, D.; Atasoylu, O.; Ozcelik, S. Investigation of StructureSpectroscopy-Function Relationship of Two-Dimensional J-Aggregates of Tetrachlorobenzimidazolocarbocyanine Preferentially Oriented in Poly-Vinyl-Alcohol Thin Films. Chem. Phys. 2009, 355, 73-80.

(11) Kobayashi, T.; Misawa, K. Hierarchical Structure of OneDimensional J-Aggregates. J. Lumin. 1997, 72-4, 38-40.

(12) Misawa, K.; Ono, H.; Minoshima, K.; Kobayashi, T. New Fabrication Method for Highly Oriented J-Aggregates Dispersed in Polymer-Films. Appl. Phys. Lett. 1993, 63, 577-579.

(13) Hirano, Y.; Okada, T. M.; Miura, Y. F.; Sugi, M.; Ishii, T. Size and Molecular Configuration of Dye Aggregates in Mixed LangmuirBlodgett Films Based on Merocyanine Dye. J. Appl. Phys. 2000, 88, 5194-5201.

(14) Ikegami, K.; Mingotaud, C.; Lan, M. B. J-Aggregates in Langmuir-Blodgett Films of a Merocyanine Dye without Metallic Cations: Using FT-IR To Observe Changes in the Electronic Structure of the Molecules Upon Aggregation. J. Phys. Chem. B 1999, 103, 1126111268.

(15) Maskasky, J. E. The Orientation of Individual J-Aggregates on Cubic $\mathrm{AgCl}$ and $\mathrm{AgBr}$ Emulsion Grains. J. Imaging Sci. 1991, 35, 29-39.

(16) Miyamoto, N.; Kuroda, K.; Ogawa, M. Uni-Directional Orientation of Cyanine Dye Aggregates on a $\mathrm{K}_{4} \mathrm{Nb}_{6} \mathrm{O}_{17}$ Single Crystal: Toward Novel Supramolecular Assemblies with Three-Dimensional Anisotropy. J. Am. Chem. Soc. 2001, 123, 6949-6950.

(17) Kinoshita, S.; Fujimoto, A.; Tomioka, A. Molecular Ordering in Self-Organized Dye Particles: Polarized Evanescent-Field Studies. J. Microsc.-Oxf. 2008, 229, 457-462.

(18) Takazawa, K.; Kitahama, Y.; Kimura, Y. Self-Oriented Pseudoisocyanine J-Aggregates in Solution. Chem. Commun. 2004, 2272-2273.

(19) Tanaka, T.; Matsumoto, S.; Kobayashi, T.; Satoh, M.; Aoyama, T. Highly Oriented J-Aggregates of Bisazomethine Dye on Aligned Poly(Tetrafluoroethylene) Surfaces. J. Phys. Chem. C 2011, 115, 19598-19605.

(20) Demir, M. M.; Ozen, B.; Ozcelik, S. Formation of Pseudoisocyanine J-Aggregates in Poly(vinyl alcohol) Fibers by Electrospinning. J. Phys. Chem. B 2009, 113, 11568-11573.

(21) Li, D.; Wang, Y. L.; Xia, Y. N. Electrospinning of Polymeric and Ceramic Nanofibers as Uniaxially Aligned Arrays. Nano Lett. 2003, 3, 1167-1171.

(22) Doshi, J.; Reneker, D. H. Electrospinning Process and Applications of Electrospun Fibers. J. Electrost. 1995, 35, 151-160.

(23) Theron, A.; Zussman, E.; Yarin, A. L. Electrostatic Field-Assisted Alignment of Electrospun Nanofibres. Nanotechnology 2001, 12, 384390.

(24) Bashouti, M.; Salalha, W.; Brumer, M.; Zussman, E.; Lifshitz, E. Alignment of Colloidal Cds Nanowires Embedded in Polymer Nanofibers by Electrospinning. ChemPhysChem 2006, 7, 102-106.

(25) Xie, X. L.; Mai, Y. W.; Zhou, X. P. Dispersion and Alignment of Carbon Nanotubes in Polymer Matrix: A Review. Mater. Sci. Eng., $R$ 2005, 49, 89-112.

(26) Roskov, K. E.; Kozek, K. A.; Wu, W. C.; Chhetri, R. K.; Oldenburg, A. L.; Spontak, R. J.; Tracy, J. B. Long-Range Alignment of Gold Nanorods in Electrospun Polymer Nano-/Microfibers. Langmuir 2011, 27, 13965-13969. 
(27) Kuo, C. C.; Tung, Y. C.; Lin, C. H.; Chen, W. C. Novel Luminescent Electrospun Fibers Prepared from Conjugated Rod-Coil Block Copolymer of Poly 2,7-(9,9-dihexylfluorene)-block-poly(methyl methacrylate). Macromol. Rapid Commun. 2008, 29, 1711-1715.

(28) Kalra, V.; Kakad, P. A.; Mendez, S.; Ivannikov, T.; Kamperman, M.; Joo, Y. L. Self-Assembled Structures in Electrospun Poly(styreneblock-isoprene) Fibers. Macromolecules 2006, 39, 5453-5457.

(29) Kim, G. M.; Wutzler, A.; Radusch, H. J.; Michler, G. H.; Simon, P.; Sperling, R. A.; Parak, W. J. One-Dimensional Arrangement of Gold Nanoparticles by Electrospinning. Chem. Mater. 2005, 17, 4949-4957.

(30) Ozcelik, S. Steady State and Picosecond Time-Resolved Photophysics of a Benzimidazolocarbocyanine Dye. J. Lumin. 2002, 96, 141-148.

(31) Fitzpatrick, R. Advanced Classical Electromagnetism; The University of Texas: Austin, 1997.

(32) McKee, M. G.; Layman, J. M.; Cashion, M. P.; Long, T. E. Phospholipid Nonwoven Electrospun Membranes. Science 2006, 311, $353-355$.

(33) Zhang, C. X.; Yuan, X. Y.; Wu, L. L.; Han, Y.; Sheng, J. Study on Morphology of Electrospun Poly(vinyl alcohol) Mats. Eur. Polym. J. 2005, 41, 423-432.

(34) Halpert, J. E.; Tischler, J. R.; Nair, G.; Walker, B. J.; Liu, W. H.; Bulovic, V.; Bawendi, M. G. Electrostatic Formation of Quantum Dot/JAggregate Fret Pairs in Solution. J. Phys. Chem. C 2009, 113, 99869992. 